\title{
The Yersinia High-Pathogenicity Island in Escherichia coli and Klebsiella pneumoniae Isolated from Polymicrobial Infections
}

\author{
RYSZARD KOCZURA*, JOANNA MOKRACKA and ADAM KAZNOWSKI \\ Department of Microbiology, Faculty of Biology, Adam Mickiewicz University, Poznań, Poland
}

Received 13 May 2011, revised 20 December 2012, accepted 21 December 2012

\begin{abstract}
We examined 12 pairs of strains of Escherichia coli and Klebsiella pneumoniae isolated from mixed infections in human for the presence of the Yersinia high-pathogenicity island (HPI). In one case both isolates carried the HPI, whereas in 11 cases one strain of the pair was HPI-positive. Although there were differences in the organization of the Yersinia HPI, all HPI-positive isolates were able to produce yersiniabactin. The presence of the Yersinia HPI may enhance the capability of strains involved in mixed infections to replicate in irondeprived conditions in the host.
\end{abstract}

Key words: HPI, mixed infections, siderophores, yersiniabactin

Iron is needed by bacteria for catalysis of DNA synthesis and for a variety of enzymes involved in electron transport and metabolism (Weinberg, 1993). In human, this element is sequestered by iron-withholding proteins such as transferrin in serum and cerebrospinal fluid, lactoferrin in cerebrospinal fluid, tears, milk, and secretions of respiratory, gastrointestinal and genital tracts. Most of the intracellular iron is bound by haem or stored in ferritin and haemosiderin (Payne, 1988). The iron-withholding mechanisms allow the host organism reducing the concentration of free iron to $10^{-18} \mathrm{M}$, which is insufficient for bacterial growth, as most bacteria, including Escherichia coli and Klebsiella pneumoniae, require $10^{-6} \mathrm{M}$ (Andrews et al., 2003). To grow and multiply under iron-deprived conditions bacteria have developed high-affinity iron acquisition systems capable of competing with the host iron-binding proteins. They consists in low-molecular-weight chelators termed siderophores, which specifically bind $\mathrm{Fe}^{3+}$ outside the cell and are subsequently taken up through receptors in the cell membrane.

In many species, strains can produce and/or use more than one siderophore (Griffiths et al., 1988). Bacterial strains can use exogenous siderophores produced by strains of other, often unrelated species (Szarapińska-Kwaszewska and Mikucki, 1999). This phenomenon is of great importance when two or more strains cause infection at the same site. Polymicrobial infections are responsible for acute and chronic diseases and are recognized with increasing frequency. They are caused by various combinations of microorganisms that interfere one with another. In synergistic polymicrobial infections, one microorganism creates a niche that facilitates infection of other microorganisms (Brogden et al., 2005).

Genes coding for siderophore-mediated iron uptake systems can be embedded in pathogenicity islands (Carniel et al., 2001; Mokracka et al., 2002). An example is yersiniabactin that has been first described in members of the genus Yersinia. Yersiniabactin-mediated iron uptake system genes are clustered in the "high-pathogenicity island" (HPI), as its presence correlates with the virulence of Yersinia spp. The Yersinia HPI has been also detected in other genera of the Enterobacteriaceae family (Bach et al., 2000; Mokracka et al., 2004).

The aim of the study was to determine the occurrence of the Yersinia high-pathogenicity island in strains of Escherichia coli and Klebsiella pneumoniae taking part in mixed infection in humans.

Twenty-four strains of E. coli and K. pneumoniae were isolated from inpatients at a hospital in Poznań, Poland. For each patient, a pair of E. coli and K. pneumoniae isolates was cultured from a single sample of urine, pharynx swab or surgical wound swab. The bacteria were identified with API 20E (bioMérieux).

The presence of the Yersinia high-pathogenicity island in K. pneumoniae isolates was screened by PCR reaction with primers irplup and irp1lp, and irp2 FP

* Corresponding author: R. Koczura, Department of Microbiology, Faculty of Biology, A. Mickiewicz University, ul. Umultowska 89, 61-614 Poznań, Poland; phone: +48 61 8295939; fax: +48 61 8295590; e-mail: koczma@amu.edu.pl 
Table I

PCR amplification of the Yersinia HPI of E. coli 10A and K. pneumoniae 10B

\begin{tabular}{|c|c|c|}
\hline \multirow{2}{*}{$\begin{array}{l}\text { Amplified HPI region (starters } \\
\text { according to Karch et al., 1999) }\end{array}$} & \multicolumn{2}{|c|}{ Amplicon size (bp) } \\
\hline & $\begin{array}{l}\text { E. coli } \\
10 \mathrm{~A}\end{array}$ & $\begin{array}{c}\text { K. pneumoniae } \\
10 \mathrm{~B}\end{array}$ \\
\hline asnT-int (asnT and int2) & 1500 & 1500 \\
\hline int (int1 and int2) & 1200 & 1200 \\
\hline int-ybtS (int5 and ybtSlp & 830 & 830 \\
\hline$y b t S$ (ybtSup and ybtSlp) & 160 & 160 \\
\hline$y b t S-y b t X-y b t Q$ (ybtSup and ybtQ3lp) & 2800 & - \\
\hline$y b t Q$ (ybtQ1 up and ybtQ1lp) & 800 & 800 \\
\hline$y b t Q-y b t P-y b t A$ (ybtQup and ybtA1lp) & 2800 & 2800 \\
\hline ybtA (ybtAup and ybtAlp) & 230 & 230 \\
\hline$y b t A$-irp2 (ybtAup and irp2 RP) & 1340 & 1340 \\
\hline irp2 (irp2 FP and irp2 RP) & 280 & 280 \\
\hline irp2-irp1 (irp2-1up and irp1-1lp) & 300 & 300 \\
\hline irp1 (irp1up and irp1lp) & 240 & 240 \\
\hline irp1-ybtU-ybtT (irp1-1up and ybtTlp) & - & - \\
\hline$y b t T-y b t E-f y u A$ (ybtTup and fyuA1lp) & 2500 & 2500 \\
\hline$y b t E-f y u A$ (ybtEup and fyuybtE) & 360 & 360 \\
\hline fyuA (fyuA FP and fyuA RP) & 780 & 780 \\
\hline
\end{tabular}

and irp2 RP specific for Y.pestis irp1 and irp2 genes, respectively. Detailed characteristics of the Yersinia HPI was done with a set of PCR reactions with primers complementary to single HPI genes as well as to regions that contained fragments of consecutive genes (Table I). PCR amplifications were done in a $25-\mu \mathrm{l}$ volume with $2 \mu \mathrm{l}$ of template DNA isolated with the boiling lysate method (Johnson and Brown, 1996), 2.5 $\mu \mathrm{l}$ of $10 \times$ PCR buffer (700 mM Tris-HCl, pH 8.6, $166 \mathrm{mM}$ $\left.\mathrm{NH}_{4}\left(\mathrm{SO}_{4}\right)^{2}, 25 \mathrm{mM} \mathrm{MgCl} 2\right), 0.25 \mu \mathrm{M}$ of each primer, $200 \mu \mathrm{M}$ of dNTP mix, and $1 \mathrm{U}$ of HiFi Taq polymerase (all reagents provided by Novazym). The PCR conditions and the sequences of primers were applied according to Karch et al. (1999). PCR products were separated in $1.5 \%$ agarose gel, stained with ethidium bromide, visualized under UV and photographed with Bio-Print V.99 system (Vilber-Lourmat, France). All experiments were performed in duplicate.

Production of yersiniabactin was detected in a crossfeeding assay (Reissbrodt and Rabsch, 1988) with Yersinia enterocolitica 5030, an indicator strain unable to produce the siderophore but capable of using exogenous yersiniabactin, and $Y$. enterocolitica 5092, a negative control strain that neither produces nor utilizes yersiniabactin (Haag et al., 1993).

It has been previously demonstrated that strains of E. coli and K. pneumoniae can harbour the Yersinia highpathogenicity island (Bach et al., 2000), a genomic island that determines production of yersiniabactin, a sidero- phore considered to be a virulence factor (Schubert etal., 2002). In the present study, we examined 24 strains of E.coli and K. pneumoniae isolated from 12 polymicrobial infections. The isolates were screened for yersiniabactin genes. In 11 cases, one strain of a pair (9 E. coli and $2 \mathrm{~K}$. pneumoniae isolates) was positive for the presence of both irp 1 and irp2 genes that code for proteins involved in yersiniabactin synthesis. In one case - E. coli 10B and K. pneumoniae 10A - both isolates harboured the HPI genes.

Our previous studies have revealed diversity of the HPI among E. coli and K. pneumoniae clinical isolates (Koczura and Kaznowski, 2003a; Koczura and Kaznowski, 2003b). To examine whether there are differences in the HPI structure of E. coli 10B and K. pneumoniae 10A strains, we performed a set of PCR reactions with primers complementary to single HPI genes as well as to regions containing the fragments of consecutive genes. The results are shown in Table I. The HPI was located in the vicinity of asnT asparagine-specific tRNA gene. Both isolates failed to give amplification product of irp1/ybtT region. PCR amplification of K. pneumoniae $10 \mathrm{~A}$ strain was also negative for $y b t S / y b t Q$ region. A difference in the structure may suggest that both strains acquired the HPI separately and not by a horizontal gene transfer between them during the infection.

To ensure that the HPI-positive isolates can produce functional yersiniabactin, we carried out biological assay with indicator strains. All strains that were positive for irp1 and irp2 genes were able to promote the growth of Y.enterocolitica 5030 indicator strain in iron-deficient conditions, whereas none of them induced the growth of Y. enterocolitica 5092 negative control. This suggests that the lack of PCR product for regions involved in yersiniabactin biosynthesis, i.e.: $y b t S$, irp 1, and $y b t T / f y u A$, were probably due to minor alterations of target sequences, as it did not affect production of the siderophore.

The ability to produce yersiniabactin is a virulence factor in Yersinia spp. (Carniel, 2001). It has been also shown that it contributes to the virulence of E. coli (Schubert et al., 2002) and K. pneumoniae (Lawlor et al., 2007). Moreover, Hancock et al. (2008) have demonstrated that E.coli strains require the yersiniabactin receptor FyuA for efficient biofilm formation, probably due to superb efficiency of yersiniabactin-mediated iron uptake system in conditions of high cell density and very low iron concentration.

Summing up, we showed that E. coli and K. pneumoniae strains involved in mixed infections can produce the same siderophore - yersiniabactin, which may enhance their survival and capability to grow in irondeprived conditions in the infected host and facilitate replication during polymicrobial infections. 


\section{Literature}

Andrews S.C., A.K. Robinson and F. Rodríguez-Quinones. 2003. Bacterial iron homeostasis. FEMS Microbiol. Rev. 27: 215-237.

Bach S., A. de Almeida and E. Carniel. 2000. The Yersinia highpathogenicity island is present in different members of the family Enterobacteriaceae. FEMS Microbiol. Lett. 183: 289-294.

Brogden K.A., J.M. Guthmiller and C. Taylor. 2005. Human polymicrobial infections. Lancet. 365: 253-55.

Carniel E. 2001. The Yersinia high-pathogenicity island: an iron uptake island. Microbes Infect. 3: 561-569.

Griffiths E., H. Chart and P. Stevenson. 1988. High-affininty iron uptake systems and bacterial virulence. pp. 121-137. In: J.A. Roth (ed.) Virulence mechanisms of bacterial pathogens. ASM Press, ashington, D.C.

Haag H., K. Hantke, H. Drechsel, I. Stojiljkovic, G. Jung and H. Zahner. 1993. Purification of yersiniabactin: a siderophore and possible virulence factor of Yersinia enterocolitica. J. Gen. Microbiol. 139: 2159-2165.

Hancock V., L. Ferriéres and P. Klemm. 2008. The ferric yersiniabactin uptake receptor FyuA is required for efficient biofilm formation by urinary tract infectious Escherichia coli in human urine. Microbiology. 154: 167-175.

Karch H., S. Schubert, D. Zhang, W. Zhang, H. Schmidt, T. Ölschläger and J. Hacker. 1999. A genomic island, termed high-pathogenicity island, is present in certain non-O157 Shiga toxin-producing Escherichia coli clonal lineages. Infect. Immun. 67: 5094-6001.
Koczura R. and A. Kaznowski. 2003a. Occurence of the Yersinia high-pathogenicity island and iron uptake systems in clinical isolates of Klebsiella pneumoniae. Microb. Pathog. 35: 196-201.

Koczura R. and A. Kaznowski. 2003b. The Yersinia high-pathogenicity island and iron uptake systems in clinical isolates of Escherichia coli. J. Med. Microbiol. 52: 637-642.

Lawlor M.S., C. O'Connor and V.L. Miller. 2007. Yersiniabactin is a virulence factor for Klebsiella pneumoniae during pulmonary infection. Infect. Immun. 75: 1463-1472.

Mokracka J., R. Koczura and A. Kaznowski. 2002. Pathogenicity islands (in Polish). Post. Mikrobiol. 41: 51-69.

Mokracka J., R. Koczura and A. Kaznowski. 2004. Yersiniabactin and other siderophores produced by clinical isolates of Enterobacter spp. and Citrobacter spp. FEMS Immunol. Med. Microbiol. 40: 51-55. Payne S.M. 1988. Iron and virulence in the family Enterobacteriaceae. CRC Crit. Rev. Microbiol. 16: 81-111.

Reissbrodt R. and W. Rabsch. 1988. Further differentiation of Enterobacteriaceae by means of siderophore-pattern analysis. $Z \mathrm{bl}$. Bakt. Hyg. A. 268: 306-317.

Schubert S., B. Picard, S. Gouriou, J. Heesemann and E. Denamur. 2002. Yersinia high-pathogenicity island contributes to virulence in Escherichia coli causing intraintestinal infections. Infect. Immun. 70: 5335-5337.

Szarapińska-Kwaszewska J. and J. Mikucki. 1999. Studies on the interchangeability of siderophores between staphylococci and various species of Gram-positive and Gram-negative bacteria (in Polish). Med. Dośw. Mikrobiol. 51: 221-232.

Weinberg E.D. 1998. Patho-ecological implications of microbial acquisition of host iron. Rev. Med. Microbiol. 9: 171-178. 